K. Yokoyama

Nagoya Math. J.

Vol. 149 (1998), 193-210

\title{
MOURRE THEORY FOR TIME-PERIODIC SYSTEMS
}

\author{
KOICHIRO YOKOYAMA
}

\begin{abstract}
Studies for A.C. Stark Hamiltonian are closely related to that for the self-adjoint operator $K=-i \frac{d}{d t}+H(t)$ on torus. In this paper we use Mourre's commutator method, which makes great progress for the study of time-independent Hamiltonian. By use of it we show the asymptotic behavior of the unitary propagator $e^{-i \sigma K}$ as $\sigma \rightarrow \pm \infty$.
\end{abstract}

\section{$\S 1$. Introduction}

We consider the following Schrödinger equation with time-dependent Hamiltonian on $\mathbb{R}^{\nu}$,

$$
\begin{gathered}
i \frac{\partial}{\partial t} u(t, x)=H(t) u(t, x), \quad(t, x) \in \mathbb{R} \times \mathbb{R}^{\nu}, \\
H(t)=-\Delta_{x}+V(t),
\end{gathered}
$$

where $V(t)$ is a multiplicative operator by a function $V(t, x)$ which is periodic in $t$ with period $2 \pi$ :

$$
V(t+2 \pi, x)=V(t, x) .
$$

As is well-known, with some suitable conditions on $V(t, x), H(t)$ generates a unique unitary propagator $\left\{U_{1}(t, s)\right\}_{-\infty<t, s<\infty}$. For $H_{0}=-\Delta_{x}$, the associated unitary propagator is denoted by $U_{0}(t, s)=e^{-i(t-s) H_{0}}$. A traditional way to study the temporal asymptotics as $t \rightarrow \pm \infty$ of $U_{1}(t, s)$ is to introduce a family of operators $\{\mathbb{U}(\sigma)\}_{\sigma \in \mathbb{R}}$ on $\mathbb{H}=L^{2}\left(\mathbb{T} \times \mathbb{R}^{\nu}\right)(\mathbb{T}=\mathbb{R} / 2 \pi \mathbb{Z})$ as follows and to investigate the asymptotic behavior of $\mathbb{U}(\sigma)$.

$$
(\mathbb{U}(\sigma) f)(t, x)=\left(U_{1}(t, t-\sigma) f(t-\sigma, \cdot)\right)(x), \quad \text { for } \quad f \in \mathbb{H} .
$$

Received May 13, 1996.

Revised January 18, 1997. 
We write the generator of this group as $-i K$. Then $K=-i \frac{d}{d t}+H(t)$ is a self-adjoint operator on $\mathbb{H}$. Let

$$
K_{0}=-i \frac{d}{d t}+H_{0}
$$

Then for short-range potentials, the wave operators

$$
\begin{aligned}
& \Omega_{ \pm}=s-\lim _{\sigma \rightarrow \pm \infty} e^{i \sigma K} e^{-i \sigma K_{0}} \quad \text { on } \quad L^{2}\left(\mathbb{T} \times \mathbb{R}^{\nu}\right) \\
& W_{ \pm}(s)=s-\lim _{t \rightarrow \pm \infty} U_{1}(t, s)^{*} U_{0}(t, s) \quad \text { on } \quad L^{2}\left(\mathbb{R}^{\nu}\right)
\end{aligned}
$$

are known to exist, and $\Omega_{ \pm}$are asymptotically complete, namely

$$
\operatorname{Ran} \Omega_{ \pm}=\mathcal{H}_{a c}(K)
$$

where $\mathcal{H}_{a c}(K)$ denotes the absolutely continuous subspace of a self-adjoint operator $K$. Moreover, the asymptotic completeness of $W_{ \pm}(s)$ holds in the following sense.

$$
\operatorname{Ran} W_{ \pm}(s)=\mathcal{H}_{a c}\left(U_{1}(s, s+2 \pi)\right) \quad \text { for all } \quad s \in \mathbb{R}
$$

These facts were first proved by Howland [How] and Yajima [Ya] by using the smoothness theory of Kato [Ka]. These results were extended to the 3-body problem by Nakamura [Na]. Kuwabara-Yajima [Ku-Y] studied the limiting absorption principle for the long-range potentials by using the pseudo-differential calculus due to Agmon and Hörmander. The asymptotic completeness of modified wave operator for long-range potential was proved by Kitada-Yajima [Ki-Y].

The aim of this paper is to accommodate the commutator technique of E. Mourre [Mo], which has brought a big progress in the spectral and scattering theory to the time-periodic 2-body Schrödinger operators. It covers almost all known results by a simpler method with weaker assumption on the potential. More precisely, we establish the limiting absorption principle for $K$ and study propagation properties of $e^{-i \sigma K}$.

Let $S$ be the set of functions $f$ such that $f \in C^{\infty}\left(\mathbb{T} \times \mathbb{R}^{\nu}\right)$ and for all $\alpha, \gamma \in \mathbb{N}$ and multi index $\beta,\left|\langle x\rangle^{\alpha} \partial_{x}^{\beta} \partial_{t}^{\gamma} f(t, x)\right| \leq C_{\alpha \beta \gamma}$ on $\mathbb{T} \times \mathbb{R}^{\nu}$ for some constant $C_{\alpha \beta \gamma}>0$. Here $\langle\cdot\rangle=\left(1+|\cdot|^{2}\right)^{\frac{1}{2}}$. As the conjugate operator $A$, which plays an important role in the Mourre theory, we adopt the following one. 
DEFINITION 1.1.

$$
A=\frac{1}{2}\left(L_{D} \cdot x+x \cdot L_{D}\right)
$$

where $\quad D_{x}=\frac{1}{i} \nabla_{x}$ and $L_{D}=\left(L_{j}\right)_{1 \leq j \leq \nu}$ with $L_{j}=D_{x_{j}}\left\langle D_{x}\right\rangle^{-2}$.

$A$ is essentially self-adjoint on domain $D=D(|x|)$. (See Theorem X.36 in $[\mathrm{R}-\mathrm{S}]$.

The following assumption is imposed on $V(t)$.

AsSUMPTION 1.2. Let $V$ be the operator of multiplication by the function $V(t, x)$ on $\mathbb{H}$. We assume that

(i) $V,[V, A]$ are extended to $K_{0}$-compact operators.

(ii) $[[V, A], A]$ is extended to a $K_{0}$-bounded operator.

We denote the extension of the form $[K, A]$ as $[K, A]^{0}$. This assumption is satisfied in the following case. The proof is given in Lemma 2.4.

EXAMPLE 1. The potential $V(t, x)$ is split into two parts $V^{L}(t, x)+$ $V^{S}(t, x)$ where $V^{L}(t, \cdot) \in C\left(\mathbb{T} ; C^{\infty}\left(\mathbb{R}^{\nu}\right)\right)$ and there exists $\delta>0$ such that

$$
\left|\partial_{x}^{\alpha} V^{L}(t, x)\right| \leq C_{\alpha}\langle x\rangle^{-\delta-|\alpha|}, \quad{ }^{\forall} \alpha .
$$

$V^{S}(t, \cdot)$ is compactly supported and $V^{S}(t, \cdot) \in C\left(\mathbb{T} ; L^{p}\left(\mathbb{R}^{\nu}\right)\right)$ with $p>$ $\max \{\nu / 2,1\}$.

Under Assumption 1.2, we have the following results.

TheOREm 1.3. Suppose Assumption 1.2 is satisfied. For $\lambda \in \mathbb{R} \backslash \mathbb{Z}$, let $d(\lambda, \mathbb{Z})$ denote the distance from $\lambda$ to $\mathbb{Z}$. Then,

(i) For all $0<\delta<d(\lambda, \mathbb{Z})$ and $f \in C_{0}^{\infty}([\lambda-\delta, \lambda+\delta])$, there exists a compact operator $\tilde{C}$ such that the following inequality holds:

$$
f(K) i[K, A]^{0} f(K) \geq \frac{2 d(I, \mathbb{Z})}{d(I, \mathbb{Z})+1} f(K)^{2}+\tilde{C},
$$

where $I=[\lambda-\delta, \lambda+\delta]$ and $d(I, \mathbb{Z})$ is the distance from $I$ to $\mathbb{Z}$. 
(ii) Eigenvalues of $K$ (the set of which are denoted by $\left.\sigma_{p p}(K)\right)$ are discrete with possible accumulation points in $\mathbb{Z}$.

If $\lambda \in \mathbb{R} \backslash\left(\mathbb{Z} \cup \sigma_{p p}(K)\right)$, for each $\epsilon>0$ there exists $0<\delta<d(\lambda, Z)$ such that

$$
f(K) i[K, A]^{0} f(K) \geq\left(\frac{2 d(I, \mathbb{Z})}{d(I, \mathbb{Z})+1}-\epsilon\right) f(K)^{2}
$$

for all $f \in C_{0}^{\infty}([\lambda-\delta, \lambda+\delta])$.

Let $\mathfrak{B}(\mathbb{H})$ be the set of bounded operators on $\mathbb{H}$.

THEOREM 1.4. Suppose $\alpha>1 / 2$.

(i) For each closed interval $I \subset \mathbb{R} \backslash\left(\mathbb{Z} \cup \sigma_{p p}(K)\right)$ the following inequalities hold:

$$
\begin{gathered}
\sup _{\operatorname{Im} z \neq 0, \operatorname{Re} z \in I}\left\|\langle A\rangle^{-\alpha}(K-z)^{-1}\langle A\rangle^{-\alpha}\right\|_{\mathfrak{B}(\mathbb{H})}<\infty, \\
\sup _{\operatorname{Im} z \neq 0, \operatorname{Re} z \in I}\left\|\langle x\rangle^{-\alpha}(K-z)^{-1}\langle x\rangle^{-\alpha}\right\|_{\mathfrak{B}(\mathbb{H})}<\infty .
\end{gathered}
$$

(ii) There exist the norm limits in $\mathfrak{B}(\mathbb{H})$.

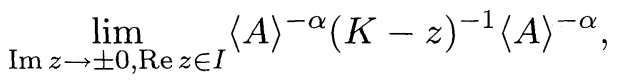

$$
\begin{aligned}
& \lim _{z \rightarrow \pm 0, \operatorname{Re} z \in I}\langle x\rangle^{-\alpha}(K-z)^{-1}\langle x\rangle^{-\alpha} .
\end{aligned}
$$

$\langle A\rangle^{-\alpha}(K-\lambda \mp i 0)^{-1}\langle A\rangle^{-\alpha}$ and $\langle x\rangle^{-\alpha}(K-\lambda \mp i 0)^{-1}\langle x\rangle^{-\alpha}$ are Hölder continuous with respect to $\lambda \in \mathbb{R} \backslash\left(\mathbb{Z} \cup \sigma_{p p}(K)\right)$.

Next we proceed to the propagation estimates. We need the following stronger assumption on the potential.

Assumption 1.5. There exists $\delta_{0}>0$ such that

$$
V(t, \cdot) \in C\left(\mathbb{T} ; C^{\infty}\left(\mathbb{R}^{\nu}\right)\right), \quad\left|\partial_{x}^{\alpha} V(t, x)\right| \leq C_{\alpha}\langle x\rangle^{-\delta_{0}-|\alpha|}, \quad{ }^{\forall} \alpha .
$$

Theorem 1.6. Suppose Assumption 1.5 is satisfied. Let $E \in \mathbb{R} \backslash(\mathbb{Z} \cup$ $\left.\sigma_{p p}(K)\right)$, and $\epsilon>0$ be given. Then there exists a small open interval $I$ containing $E$ such that for any $f \in C_{0}^{\infty}(I)$ and $s^{\prime}>s>0$,

$$
\begin{aligned}
\left\|\chi\left(\frac{|x|^{2}}{4 \sigma^{2}}-\frac{d(I, \mathbb{Z})}{d(I, \mathbb{Z})+1}<-\epsilon\right) e^{-i \sigma K} f(K)\langle x\rangle^{-s^{\prime}}\right\|_{\mathfrak{B}(\mathbb{H})} & \\
=O\left(\sigma^{-s}\right) \text { as } & \sigma \rightarrow \infty
\end{aligned}
$$


where $\chi(x<a)$ denotes the characteristic function of the interval $(-\infty, a)$.

\section{$\S 2$. Conjugate operator}

We shall assume Assumption 1.2 throughout this section. We prove the following Lemma at first.

Lemma 2.1. Let $A$ be as in 1.6. Then $e^{i A \alpha}$ leaves $D(K)$ invariant, i.e. for each $\Psi \in \mathbb{H}$

$$
\sup _{|\alpha|<1}\left\|K e^{i A \alpha}(K+i)^{-1} \Psi\right\|_{\mathbb{H}}<\infty
$$

Proof. As $V$ is $K_{0}$-compact, it is sufficient to show $e^{i A \alpha}$ leaves $D\left(K_{0}\right)$ invariant. Let $\mathfrak{F}$ be the Fourier transformation with respect to $x$, and we define $\hat{A}$ by

$$
\hat{A}=\mathfrak{F} A \mathfrak{F}^{-1} .
$$

Then $e^{i \hat{A} \alpha}$ can be expressed as

$$
\left(e^{i \hat{A} \alpha} \psi\right)(t, p)=\left|\operatorname{det}\left(\frac{\partial \Gamma_{\alpha}^{l}}{\partial p_{j}}(p)\right)\right|^{\frac{1}{2}} \psi\left(t, \Gamma_{\alpha}(p)\right),
$$

where $\Gamma_{\alpha}(p)=\left(\Gamma_{\alpha}^{l}(p)\right)_{1 \leq l \leq \nu}$ is the solution of the following differential equation

$$
\left\{\begin{array}{l}
\frac{d}{d \alpha} \Gamma_{\alpha}(p)=\left(1+\left|\Gamma_{\alpha}(p)\right|^{2}\right)^{-1} \Gamma_{\alpha}(p), \\
\Gamma_{0}(p)=p .
\end{array}\right.
$$

We note $-i \frac{d}{d t}$ on $L^{2}(\mathbb{T})$ has eigenvalues $k \in \mathbb{Z}$. Let $P_{k}$ be the associated eigenprojection. Then $K_{0}$ can be decomposed as

$$
K_{0}=\sum_{k \in \mathbb{Z}}\left(k+H_{0}\right) \otimes P_{k} .
$$

And for each $\Psi \in \mathbb{H}$

$$
\begin{aligned}
K_{0} e^{i A \alpha} & \left(K_{0}+i\right)^{-1} \Psi \\
& =\mathfrak{F}^{-1}\left(\sum_{k \in \mathbb{Z}}\left|\operatorname{det}\left(\frac{\partial \Gamma_{l}}{\partial p_{j}}\right)\right|^{\frac{1}{2}}\left(k+|p|^{2}+i\right)\left(k+\left|\Gamma_{\alpha}(p)\right|^{2}+i\right)^{-1} \otimes P_{k} \mathfrak{F} \Psi\right)
\end{aligned}
$$

From (2.4) it is easily seen that $\left.|| \Gamma_{\alpha}(p)\right|^{2}-|p|^{2}|\leq 2| \alpha \mid$, which proves the Lemma. 
Once we have proved Lemma 2.1, we can trace the Mourre theory in the same way.

Lemma 2.2. For $K$ and $A$ defined above, the following facts hold.

(i) $(K-z)^{-1}$ leaves $D(A)$ invariant for all $z \in \mathbb{C} \backslash \sigma(K)$.

(ii) $(A+i \lambda)^{-1}$ leaves $D(K)$ invariant for all $\lambda \in \mathbb{R}$, and $\lim _{|\lambda| \rightarrow \infty}(K+i) i \lambda(A+i \lambda)^{-1}(K+i)^{-1} \Psi=\Psi$ for all $\Psi \in \mathbb{H}$.

Corollary 2.3. (the Virial theorem) For all $\Psi \in D(K)$, $\lim _{|\lambda| \rightarrow \infty} i\left[K, i \lambda A(A+i \lambda)^{-1}\right] \Psi=i[K, A]^{0} \Psi$.

For the proof of Lemma 2.2 and Corollary 2.3, see [Mo].

Proof of Theorem 1.3. By the symbol calculus we have

$$
\begin{aligned}
i[K, A] & =i\left[H_{0}, A\right]+i[V, A] \\
& =2 H_{0}\left(H_{0}+1\right)^{-1}+i[V, A] .
\end{aligned}
$$

Let us recall the well-known formula of functional calculus [H-S]. Let $f \in C^{\infty}(\mathbb{R})$ be such that for some $m_{0} \in \mathbb{R}$

$$
\left|f^{(k)}(t)\right| \leq C_{k}(1+|t|)^{m_{0}-k}, \quad \forall k \in \mathbb{N} \cup\{0\}
$$

Then we can construct an almost analytic extension $\tilde{f}(z)$ of $f(t)$ satisfying

$$
\begin{aligned}
& \tilde{f}(t)=f(t), \quad t \in \mathbb{R}, \\
& \left|\partial_{\bar{z}} \tilde{f}\right| \leq C_{N}|\operatorname{Im} z|^{N}\langle z\rangle^{m_{0}-1-N}, \quad{ }^{\prime} N \in \mathbb{N}, \\
& \operatorname{supp} \tilde{f}(z) \subset\{z ;|\operatorname{Im} z| \leq 1+|\operatorname{Re} z|\} .
\end{aligned}
$$

We remark that supp $\tilde{f}$ is compact in $\mathbb{C}$ if $f \in C_{0}^{\infty}(\mathbb{R})$ (due to Appendix in [Gé1]).

Further, if (2.5) holds with $m_{0}<0$ we have

$$
f(K)=\frac{1}{2 \pi i} \int_{\mathbb{C}} \partial_{\bar{z}} \tilde{f}(z)(z-K)^{-1} d z \wedge d \bar{z} .
$$


We assume $\lambda \in(l, l+1)$ with some $l \in \mathbb{N}$. From Assumption 1.2 and the above formula, $f(K)-f\left(K_{0}\right)$ is a compact operator. Therefore we have

$$
\begin{aligned}
f(K) i[K, A]^{0} f(K) & =2 f(K) H_{0}\left(H_{0}+1\right)^{-1} f(K)+f(K) i[V, A]^{0} f(K) \\
& =2 f\left(K_{0}\right) H_{0}\left(H_{0}+1\right)^{-1} f\left(K_{0}\right)+\text { (compact operator). }
\end{aligned}
$$

By decomposing $K_{0}$ as $\sum_{k \in \mathbb{Z}}\left(k+H_{0}\right) \otimes P_{k}$ again

$$
2 f\left(K_{0}\right) H_{0}\left(H_{0}+1\right)^{-1} f\left(K_{0}\right)=2 \sum_{k \in \mathbb{Z}} H_{0}\left(H_{0}+1\right)^{-1} f\left(k+H_{0}\right)^{2} \otimes P_{k}
$$

Since supp $f(k+\cdot) \subset[\lambda-\delta-k, \lambda+\delta-k]$ and $\frac{t}{t+1}$ is a monotone increasing function for $t \geq 0$, we have the following inequality

$$
\begin{aligned}
f\left(K_{0}\right) H_{0}\left(H_{0}+1\right)^{-1} f\left(K_{0}\right) & \geq \sum_{k \leq l} \frac{\lambda-\delta-l}{\lambda-\delta-l+1} f\left(k+H_{0}\right)^{2} \otimes P_{k} \\
& \geq \frac{d(I, \mathbb{Z})}{d(I, \mathbb{Z})+1} f\left(K_{0}\right)^{2},
\end{aligned}
$$

which proves(1). By shrinking supp $f$ we also obtain (2).

We omit the proof of Theorem 1.4. Since it follows from Theorem 1.3 by the well-known arguments.

Lemma 2.4. Let $V(t, x)$ be as in Example 1. Then as a multiplicative operator, $V=V(t, x)$ satisfies Assumption 1.2.

Proof. As was proved by Yajima (Lemma 3.1 in [Ya]), if $W(t, x) \in$ $C\left(\mathbb{T} ; L^{p}\left(\mathbb{R}^{\nu}\right)\right)$ with $p>\max \{\nu / 2,1\}, W$ is $K_{0}$-compact. $K_{0}$-compactness of $\left[V^{s}, A\right]$ and $K_{0}$-boundness of $\left[\left[V^{s}, A\right], A\right]$ also hold as we take $V^{s}(t, x)$ supported in a compact set. One can also see the following fact: For any $\delta>0$, $\langle x\rangle^{-\delta}$ is a $K_{0}$-compact operator. In fact, we have only to approximate $\langle x\rangle^{-\delta}$ by a compactly supported function. It indicates that $V^{L}$ is $K_{0}$-compact. For the sake of convenience, we write $V$ and $D_{j}$ instead of $V^{L}$ and $D_{x_{j}}$. It is sufficient to show that $\left[V, X_{j} L_{j}\right]$ is $K_{0}$-compact, and $\left[\left[V, X_{j} L_{j}\right], X_{k} L_{k}\right]$ is $K_{0}$-bounded. Here $1 \leq j, k \leq \nu$ and $X_{j}$ is a multiplicative operator by a function $x_{j}$. We denote $x_{j} V(t, x)$ as $V_{j}(t, x)$. At first we split the commutator into two parts

$$
\begin{aligned}
{\left[V, X_{j} L_{j}\right] } & =\left[V_{j}, L_{j}\right]+\left[L_{j}, X_{j}\right] V \\
& \equiv I_{1}+I_{2} .
\end{aligned}
$$


From the assumption we assume in Example 1, we can easily see that $I_{2}\langle x\rangle^{\delta} \in \mathfrak{B}(\mathbb{H})$. For $I_{1}$, we split it again

$$
\begin{aligned}
I_{1} & =\left\langle D_{x}\right\rangle^{-2}\left\{H_{0} V_{j} D_{j}-D_{j} V_{j} H_{0}\right\}\left\langle D_{x}\right\rangle^{-2}+\left\langle D_{x}\right\rangle^{-2}\left[V_{j}, D_{j}\right]\left\langle D_{x}\right\rangle^{-2}, \\
& \equiv I_{3}+I_{4} .
\end{aligned}
$$

We use the Assumption for $V^{L}$ to see $I_{4}\langle x\rangle^{1+\delta} \in \mathfrak{B}(\mathbb{H})$. We can rewrite $I_{3}$ as

$$
\left\langle D_{x}\right\rangle^{-2}\left\{\left(-\Delta V_{j}\right) D_{j}-2\left(\left(\nabla V_{j}\right) \cdot \nabla\right) D_{j}+\left[V_{j}, D_{j}\right] H_{0}\right\}\left\langle D_{x}\right\rangle^{-2} .
$$

We use the Assumption for $V^{L}$ again to prove that $\left[V^{L}, A\right]$ is $K_{0}$-compact. As for the double commutator, we compute

$$
\left[\left[V, X_{j} L_{j}\right], X_{k} L_{k}\right]=\left[I_{2}+I_{3}+I_{4}, X_{k} L_{k}\right]
$$

We can easily obtain the following result by using the pseudo differential calculus, as we commute $X_{k} D_{k}$ with $V$ or another PsDO.

$$
\left[I_{\alpha}, X_{k} L_{k}\right] \text { is } K_{0} \text {-compact for } \alpha=2,3,4 \text {. }
$$

\section{$\S 3$. Propagation estimate}

We shall prove Theorem 1.6 in this section. We follow the abstract framework constructed by Skibsted [Sk]. In our case $K$ is not a semibounded operator, which introduces a slight difference in applying the method of $[\mathrm{Sk}]$. From Assumption 1.5, it follows that $[K, A]$ is extended to a bounded operator. We add this condition as an additional assumption in the abstract framework.

Definition 3.1. Given $\beta, \alpha \geq 0$ and $\epsilon>0$, we denote by $\mathfrak{F}_{\beta, \alpha, \epsilon}$ as the set of function $g$ of the form, $g(x, \tau)=g_{\beta, \alpha, \epsilon}(x, \tau)=-\tau^{-\beta}(-x)^{\alpha} \chi\left(\frac{x}{\tau}\right)$ defined for $(x, \tau) \in \mathbb{R} \times \mathbb{R}^{+}$, where $\chi \in C^{\infty}(\mathbb{R})$ and satisfies the following properties:

$$
\begin{aligned}
& \chi(x)=1 \text { for } x<-2 \epsilon, \chi(x)=0 \text { for } x>-\epsilon . \\
& \frac{d}{d x} \chi(x) \leq 0 \text { and } \alpha \chi(x)+x \frac{d}{d x} \chi(x)=\tilde{\chi}(x)^{2} \text { for some } \tilde{\chi} \in C^{\infty}(\mathbb{R}), \tilde{\chi} \geq 0
\end{aligned}
$$


It follows from the last equation that $\left(g^{(1)}(x, \tau)\right)^{\frac{1}{2}}$ is smooth. Here $g^{(n)}(x, \tau)$ $=(\partial / \partial x)^{n} g(x, \tau)$. For operators $P$ and $Q$, we define $\operatorname{ad}_{Q}^{0}(P)=P$ and for $m \in \mathbb{N}, \operatorname{ad}_{Q}^{m}(P)=\left[\operatorname{ad}_{Q}^{m-1}(P), Q\right]$ inductively.

Lemma 3.2. Let $A$ and $P$ be linear operators on $\mathbb{H}$. Suppose $A$ is self-adjoint and P-bounded. Suppose that the form $\operatorname{ad}_{A}^{m}(P)$ extends to a bounded operator for $1 \leq m \leq n$. Then for any $g \in C^{\infty}(\mathbb{R})$ satisfying 2.5 with $m_{0}<n$

(i)

$$
P g(A)=\sum_{m=0}^{n-1} \frac{g^{(m)}(A)}{m !} \operatorname{ad}_{A}^{m}(P)+\frac{1}{2 \pi i} \int_{\mathbb{C}} \partial_{\bar{z}} \tilde{g}(z) R_{n, A, P}^{r}(z) d z \wedge d \bar{z},
$$

where $R_{n, A, P}^{r}(z)=(z-A)^{-n} \operatorname{ad}_{A}^{n}(P)(z-A)^{-1}$.

(ii)

$$
g(A) P=\sum_{m=0}^{n-1} \operatorname{ad}_{A}^{m}(P) \frac{(-1)^{m}}{m !} g^{(m)}(A)+\frac{1}{2 \pi i} \int_{\mathbb{C}} \partial_{\bar{z}} \tilde{g}(z) R_{n, A, P}^{l}(z) d z \wedge d \bar{z},
$$

where $R_{n, A, P}^{l}(z)=(z-A)^{-1} \operatorname{ad}_{A}^{n}(P)(A-z)^{-n}$ and $\tilde{g}(z)$ denotes an almost analytic extension of $g(x)$.

These formulas of asymptotic expansion are obtained by virtue of (2.6) and the calculus of the commutator $\left[(z-A)^{-1}, P\right]$. (See Lemma 3.3 in [Gé2].)

Assumption 3.3. Let $n_{0} \in \mathbb{N}, \sigma_{0}>0, n_{0}-\frac{1}{2}>\alpha_{0}>0$. Let $f$, $f_{2} \in C_{0}^{\infty}(\mathbb{R})$ be such that $f_{2} f=f$ and $K, A(\tau), B$ be self-adjoint operators on $\mathbb{H}$. Assume that $A(\tau)$ have common domain $D$ for $\tau=\sigma+\sigma_{0} \sigma \geq 0$, $D(K) \cap D$ is dense in $D(K), B \geq I$ and that $\left\langle A_{0}\right\rangle^{\frac{n_{0}}{2}}\langle B\rangle^{-\frac{n_{0}}{2}} \in \mathfrak{B}(\mathbb{H})$ with $A_{0}=A\left(\sigma_{0}\right)$. Assume moreover

(i) With $1 \leq n \leq n_{0}$, $i^{n} \operatorname{ad}_{A(\tau)}^{n}(K)$ extends to a bounded self-adjoint operator, and $\operatorname{ad}_{A(\tau)}^{n}(K)=O(1)$ in $\mathfrak{B}(\mathbb{H})$ as $\tau \rightarrow \infty$.

(ii) If $A(\tau)$ is unbounded, $\sup _{|\alpha|<1}\left\|K e^{i A(\tau) \alpha} \psi\right\|_{\mathbb{H}}<\infty$ for any $\psi \in D(K)$ and $\tau \geq \sigma_{0}$. 
(iii) For each $\tau_{1}, \tau_{2} \geq \sigma_{0}, A\left(\tau_{1}\right)-A\left(\tau_{2}\right)$ is a bounded operator, and the derivative $d_{\tau} A(\tau)=\frac{d}{d \tau} A(\tau)$ exists in $\mathfrak{B}(\mathbb{H})$. Further $\operatorname{ad}_{A(\tau)}^{n-1}\left(d_{\tau} A(\tau)\right)=$ $O(1)$ in $\mathfrak{B}(\mathbb{H})$ as $\tau \rightarrow \infty$ for $1 \leq n \leq n_{0}$.

(iv) For $n \leq n_{0} \operatorname{ad}_{A(\tau)}^{n}(K)$ and $\operatorname{ad}_{A(\tau)}^{n-1}\left(d_{\tau} A(\tau)\right)$ are continuous $\mathfrak{B}(\mathbb{H})$-valued functions of $\tau \geq \sigma_{0}$.

(v) There exists $\delta>0$ such that the following condition $q\left(\beta_{0}, \alpha_{0}, \delta\right)$ holds. $q\left(\beta_{0}, \alpha_{0}, \delta\right)$ : Let $D A(\tau)$ denote the symmetric operator $i[K, A(\tau)]+$ $d_{\tau} A(\tau)$.

There exist bounded operators $B_{1}(\tau)$ and $B_{2}(\tau)$ on $\mathbb{H}$ such that

$$
f_{2}(K) D A(\tau) f_{2}(K) \geq B_{1}(\tau)+B_{2}(\tau)
$$

$\left\|B_{1}(\tau)\right\|_{\mathfrak{B}(\mathbb{H})}=O\left(\tau^{-\delta}\right)$ as $\tau \rightarrow \infty$, and for $(\beta, \alpha)=(0,1), \ldots,\left(0, \alpha_{0}^{\prime}\right)$, $\left(\beta_{0}, \alpha_{0}\right)\left(\alpha_{0}^{\prime}=\max \left\{m \in \mathbb{N}: m<\alpha_{0}\right\}\right)\left(=\left(\beta_{0}, \alpha_{0}\right)\right.$ if $\left.\alpha_{0}<1\right)$ the following estimates holds:

Given $\epsilon>0$ and $g(x, \tau) \in \mathfrak{F}_{\beta, \alpha, \epsilon}$, there exists $C>0$ such that with $\zeta(\sigma)=\left(g^{(1)}(A(\tau), \tau)\right)^{\frac{1}{2}} e^{-i \sigma K} f(K) B^{-\frac{\alpha}{2}} \phi$

$$
\int_{0}^{\infty} d \sigma\left|\left(\zeta(\sigma), B_{2}(\tau) \zeta(\sigma)\right)_{\mathbb{H}}\right| \leq C\|\phi\|^{2}, \quad{ }^{\forall} \phi \in \mathbb{H},
$$

where $(\cdot, \cdot)_{\mathbb{H}}=(\cdot, \cdot)$ is the inner product of $\mathbb{H}$.

THEOREM 3.4. Suppose Assumption 3.3 is satisfied and in addition,

$$
\begin{aligned}
\alpha_{0}+2 & <\beta_{0}+n_{0}, \\
\alpha_{0}^{\prime}+2 & <n_{0}, \quad\left(\text { if } \quad \alpha_{0}>1\right) \\
\frac{\alpha_{0}}{2}+\frac{5}{2} & <n_{0}+\beta_{0}, \\
\alpha_{0}^{\prime}+\frac{5}{2} & \leq n_{0}, \quad\left(\text { if } \quad \alpha_{0}>1\right) .
\end{aligned}
$$

Then for $(\beta, \alpha)=(0,1), \ldots,\left(0, \alpha_{0}^{\prime}\right),\left(\beta_{0}, \alpha_{0}\right)\left(=\left(\beta_{0}, \alpha_{0}\right)\right.$ if $\left.\alpha_{0}<1\right)$, any $\epsilon>0$ and $g(x, \tau) \in \mathfrak{F}_{\beta, \alpha, \epsilon}$,

$$
\left\|\left(-g_{\beta, \alpha, \epsilon}(A(\tau), \tau)\right)^{\frac{1}{2}} e^{-i \sigma K} f(K) B^{-\frac{\alpha}{2}}\right\|_{\mathcal{B}(\mathbb{H})}=O(1) \quad \text { as } \quad \tau \rightarrow \infty
$$


COROLlary 3.5. Under the same conditions in Theorem 3.4, we have the following result:

For $(\beta, \alpha)=(0,1), \ldots,\left(0, \alpha_{0}^{\prime}\right),\left(\beta_{0}, \alpha_{0}\right)$, any $\epsilon>0, g(x, \tau) \in \mathfrak{F}_{\beta, \alpha, \epsilon}$, and $1 \geq \theta \geq 0$

$$
\begin{aligned}
\left\|\left(-g_{0, \alpha(1-\theta), \epsilon}(A(\tau), \tau)\right)^{\frac{1}{2}} e^{-i \sigma K} f(K) B^{-\frac{\alpha}{2}}\right\|_{\mathfrak{B}(\mathbb{H})} & \\
& =O\left(\tau^{(\beta-\alpha \theta) / 2}\right) \quad \text { as } \quad \tau \rightarrow \infty .
\end{aligned}
$$

We note that (3.7) is easily obtained by (3.6) and the inequality

$$
-\tau^{-\beta}(\epsilon \tau)^{\alpha \theta} g_{0, \alpha(1-\theta), 2 \epsilon}(x, \tau) \leq-g_{\beta, \alpha, \epsilon}(x, \tau) .
$$

Sketch of Proof. The proof of Theorem 3.4 is almost the same as that of Theorem 2.4 in $[\mathrm{Sk}]$. Let $f_{1} \in C_{0}^{\infty}(\mathbb{R})$ be real valued and satisfy $f_{1} f_{2}=f_{2}$. We denote $\psi(\sigma)=e^{-i \sigma K} f(K) B^{-\alpha / 2} \phi$, and $D_{1} A(\tau)=d_{\tau} A(\tau)+i\left[f_{1}(K) K\right.$, $A(\tau)]$. Then $(\psi(\sigma), g(A(\tau), \tau) \psi(\sigma))$ is continuously differentiable with

$$
\frac{d}{d \sigma}(\psi(\sigma), g(A(\tau), \tau) \psi(\sigma))=(\psi(\sigma), D g(A(\tau), \tau) \psi(\sigma))
$$

where

$$
\begin{array}{r}
D g(A(\tau), \tau)=\left(\frac{\partial}{\partial \tau} g\right)(A(\tau), \tau)+\sum_{m=1}^{n_{0}-1}(m !)^{-1} g^{(m)}(A(\tau), \tau) \operatorname{ad}_{A(\tau)}^{m-1}\left(D_{1} A(\tau)\right) \\
\quad+\frac{1}{2 \pi i} \int_{\mathbb{C}} \partial_{\bar{z}} \tilde{g}(z, \tau)(z-A(\tau))^{-n_{0}} \operatorname{ad}_{A(\tau)}^{n_{0}-1}\left(D_{1} A(\tau)\right)(z-A(\tau))^{-1} d z \wedge d \bar{z}
\end{array}
$$

We can then prove that $(\psi(\sigma), D g(A(\tau), \tau) \psi(\sigma))$ is integrable with respect to $\tau$, which indicates the assertion of Theorem 3.4. Corollary 3.5 follows from the same argument as in $[\mathrm{Sk}]$.

With these results, we proceed to prove the propagation estimate for operator $K$ with potential $V$ satisfying Assumption 1.5.

Suppose $E \in \mathbb{R} \backslash \mathbb{Z}$ and $0<E^{\prime}<\frac{d(E, \mathbb{Z})}{d(E, \mathbb{Z})+1}$. We choose $f$ and $f_{2}$ as in Assumption 3.3, with support in a small interval $I \subset \mathbb{R} \backslash \mathbb{Z}$. Put $\sigma_{0}=1$, $A_{1}(\tau)=A-2 E^{\prime} \tau(\tau=\sigma+1)$, and $B=\left\langle A_{1}(1)\right\rangle$.

By virtue of Lemma 2.1 and some elementary calculus one can prove that $A_{1}(\tau)$ verifies Assumption 3.3 with arbitrary $n_{0}, \alpha_{0}, \beta_{0}$. By the same argument as in the proof of Corollary 3.5 we have that:

$$
\left\|\left(-\frac{A_{1}(\tau)}{\tau}\right)^{\frac{1}{2}} \chi\left(\frac{A_{1}(\tau)}{\tau}\right) e^{-i \sigma K} f(K) B^{-s}\right\|_{\mathfrak{B}(\mathbb{H})}=O\left(\tau^{-s}\right) \text { as } \tau \rightarrow \infty
$$


for $s \geq \frac{1}{2}$.

LEMMA 3.6. Fix $0<E^{\prime \prime}<E^{\prime}<\frac{d(E, \mathbb{Z})}{d(E, \mathbb{Z})+1}$. Let $f_{2}, f, \sigma_{0}, \beta_{0}$ and $\alpha_{0}$ as above. For an arbitrary fixed $\epsilon^{\prime \prime}>0$ we take $g \in \mathfrak{F}_{0,1, \epsilon^{\prime \prime}}$ satisfying $(-g(x, \tau))^{\frac{1}{2}},\left(-\left(\frac{\partial}{\partial \tau} g\right)(x, \tau)\right)^{\frac{1}{2}} \in C^{\infty}\left(\mathbb{R} \times \mathbb{R}^{+}\right)$. We put $M(x, \xi)=\left(E^{\prime \prime}-\right.$ $\left.\frac{|x|^{2}}{4\langle\xi\rangle^{2}}\right)^{\frac{1}{2}}, G=\left(-g\left(-\tau M\left(\frac{x}{\tau}, \xi\right), \tau\right)\right)_{\mid \xi=D_{x}}^{\frac{1}{2}}$ and set $A_{2}(\tau)=-G^{*} G$

Then for all $\beta_{0}, \alpha_{0}, n_{0}$, there exists $\delta>0$ such that $A_{2}(\tau)$ satisfies Assumption 3.3.

Before the proof of this Lemma, we introduce a symbol class and asymptotic expansion formulas.

Definition 3.7. For $l, m \in \mathbb{R}$, let $S\left(\tau^{l}\langle\xi\rangle^{m}\right)$ be the set of functions $a_{\tau}(x, \xi) \in C^{\infty}\left(\mathbb{R}_{x}^{\nu} \times \mathbb{R}_{\xi}^{\nu}\right)$ such that

$$
\left|\partial_{x}^{\alpha} \partial_{\xi}^{\beta} a_{\tau}(x, \xi)\right| \leq C_{\alpha \beta} \tau^{l-|\alpha|}\langle\xi\rangle^{m-|\beta|}, \quad(x, \xi) \in \mathbb{R}_{x}^{\nu} \times \mathbb{R}_{\xi}^{\nu}
$$

for all multi-indexes $\alpha, \beta$.

We write $a_{\tau}\left(x, D_{x}\right) \in \operatorname{Op} S\left(\tau^{l}\left\langle D_{x}\right\rangle^{m}\right)$, if $a_{\tau}(x, \xi) \in S\left(\tau^{l}\langle\xi\rangle^{m}\right)$.

LEMmA 3.8. Suppose $a_{\tau}(x, \xi) \in S\left(\tau^{l}\langle\xi\rangle^{m}\right)$, and $b_{\tau}(x, \xi) \in S\left(\tau^{l^{\prime}}\langle\xi\rangle^{m^{\prime}}\right)$. Then $a_{\tau}\left(x, D_{x}\right)^{*} \in \mathrm{Op} S\left(\tau^{l}\left\langle D_{x}\right\rangle^{m}\right)$ and $a_{\tau}\left(x, D_{x}\right) b_{\tau}\left(x, D_{x}\right) \in \mathrm{Op} S\left(\tau^{l+l^{\prime}}\right.$ $\left.\left\langle D_{x}\right\rangle^{m+m^{\prime}}\right)$. We have the following asymptotic formulas.

$$
a_{\tau}\left(x, D_{x}\right)^{*}-\sum_{|\alpha|<N} \frac{1}{\alpha !}{\overline{a_{\tau}}}_{(\alpha)}^{(\alpha)}(x, \xi)_{\mid \xi=D_{x}} \in \mathrm{Op} S\left(\tau^{l-N}\left\langle D_{x}\right\rangle^{m-N}\right)
$$

where $p_{(\beta)}^{(\alpha)}(x, \xi)=D_{\xi}^{\alpha} \partial_{x}^{\beta} p(x, \xi)$,

$$
\begin{aligned}
a_{\tau}\left(x, D_{x}\right) b_{\tau}\left(x, D_{x}\right)-\sum_{|\alpha|<N} \frac{1}{\alpha !} a_{\tau}^{(\alpha)}(x, \xi) b_{\tau(\alpha)}(x, \xi)_{\mid \xi=D_{x}} & \\
& \in \mathrm{Op} S\left(\tau^{l+l^{\prime}-N}\left\langle D_{x}\right\rangle^{m+m^{\prime}-N}\right) .
\end{aligned}
$$

Proof of Lemma 3.6. We rewrite $D A_{2}(\tau)=-\left(d_{\tau} G\right)^{*} G-G^{*}\left(d_{\tau} G\right)+$ $i\left[K, A_{2}(\tau)\right]$. Let $M$ denote $M\left(\frac{x}{\tau}, \xi\right)$. It can be easily verified that $G \in S\left(\tau^{\frac{1}{2}}\right)$ and

$$
\begin{array}{r}
\left(d_{\tau} G\right)^{*} G=-\frac{1}{2}\left\{\left(\frac{\partial}{\partial \tau} g\right)(-\tau M, \tau)-g^{(1)}(-\tau M, \tau) E^{\prime \prime} M^{-1}\right\} \mid \xi=D_{x} \\
+\operatorname{Op} S\left(\tau^{-1}\right)
\end{array}
$$


Using the assumption for $g$ in Lemma 3.6, we have

$$
\begin{aligned}
-\left(\frac{\partial}{\partial \tau} g\right)(-\tau M, \tau)_{\mid \xi=D_{x}}=\left\{\left(-\frac{\partial}{\partial \tau} g\right)_{\mid \xi=D_{x}}^{\frac{1}{2}}\right\}^{*}\left\{\left(-\frac{\partial}{\partial \tau} g\right)_{\mid \xi=D_{x}}^{\frac{1}{2}}\right\} & \\
& +\operatorname{Op} S\left(\tau^{-1}\right)
\end{aligned}
$$

The last term $i\left[K, A_{2}(\tau)\right]$ has the following expression

$$
\begin{aligned}
i\left[K, A_{2}(\tau)\right]=\left\{g^{(1)}(-\tau M, \tau) M^{-1} \cdot \frac{1}{2 \tau} \frac{x \cdot \xi}{\langle\xi\rangle^{2}}\right\}_{\mid \xi=D_{x}} & \\
& +i\left[V, A_{2}(\tau)\right]+\operatorname{Op} S\left(\tau^{-1}\right)
\end{aligned}
$$

We denote $\left(g^{(1)}(-\tau M, \tau) M^{-1}\right)^{\frac{1}{2}} \mid \xi=D_{x}$ as $g_{H}\left(x, D_{x}\right) \in \mathrm{Op} S(1)$. We also remark that $\frac{1}{\tau} \frac{x \cdot \xi}{\langle\xi\rangle^{2}} g_{H}(x, \xi) \in S(1)$. We can rewrite the right hand side of (3.14) as

$$
\frac{1}{2} g_{H}\left(x, D_{x}\right)^{*}\left(\frac{A_{1}(\tau)}{\tau}+2 E^{\prime}\right) g_{H}\left(x, D_{x}\right)+i\left[V, A_{2}(\tau)\right]+R_{0}(\tau)
$$

where $\left\|R_{0}(\tau)\right\|_{\mathfrak{B}(\mathbb{H})}=O\left(\tau^{-1}\right)$ as $\tau \rightarrow \infty$.

For $i\left[V, A_{2}(\tau)\right]$, we obtain $\left\|\left[V, A_{2}(\tau)\right]\right\|_{\mathfrak{B}(\mathbb{H})}=O\left(\tau^{-\delta_{0}}\right)$ by computing $\nabla_{x} V^{L}$. $\nabla_{\xi}(g(-\tau M, \tau))$

Summing up, we have

$$
D A_{2}(\tau) \geq \frac{1}{2} g_{H}\left(x, D_{x}\right)^{*}\left(\frac{A_{1}(\tau)}{\tau}+2\left(E^{\prime}-E^{\prime \prime}\right)\right) g_{H}\left(x, D_{x}\right)+R_{1}(\tau)
$$

where $\delta_{1}=\min \left\{\delta_{0}, 1\right\}$ and $\left\|R_{1}(\tau)\right\|_{\mathfrak{B}(\mathbb{H})}=O\left(\tau^{-\delta_{1}}\right)$ as $\tau \rightarrow \infty$.

Since

$$
\frac{A_{1}(\tau)}{\tau}+2\left(E^{\prime}-E^{\prime \prime}\right) \geq \frac{A_{1}(\tau)}{\tau} \chi\left(\frac{A_{1}(\tau)}{\tau}\right),
$$

we can replace $\frac{A_{1}(\tau)}{\tau}+2\left(E^{\prime}-E^{\prime \prime}\right)$ by $\frac{A_{1}(\tau)}{\tau} \chi\left(\frac{A_{1}(\tau)}{\tau}\right)$ with $\epsilon=E^{\prime}-E^{\prime \prime}$. Thus it suffices to prove $\left(-\frac{A_{1}(\tau)}{\tau}\right)^{1 / 2} \chi\left(\frac{A_{1}(\tau)}{\tau}\right) g_{H}\left(x, D_{x}\right) f_{2}(K)\left(g_{\beta, \alpha, \epsilon}^{(1)}\left(A_{2}(\tau), \tau\right)\right)^{1 / 2}$ $e^{-i \sigma K} f(K) B^{-\alpha / 2}$ is square integrable.

For $l \in \mathbb{N} \cup\{0\}$, we put $g_{l}(x, \tau)=\left(\frac{\partial}{\partial x}\right)^{l}\left(\left(-\frac{x}{\tau}\right)^{\frac{1}{2}} \chi\left(\frac{x}{\tau}\right)\right)$ and we write the 
almost analytic extension of $g_{l}(x, \tau)$ as $\tilde{g}_{l}(z, \tau)$. From (3.2)

$$
\begin{aligned}
\left(-\frac{A_{1}(\tau)}{\tau}\right)^{\frac{1}{2}} & \chi\left(\frac{A_{1}(\tau)}{\tau}\right) g_{H}\left(x, D_{x}\right) \\
= & \sum_{m=0}^{n_{0}-1} \frac{(-1)^{m}}{m !} \operatorname{ad}_{A_{1}(\tau)}^{m}\left(g_{H}\left(x, D_{x}\right)\right) g_{0}^{(m)}\left(A_{1}(\tau), \tau\right) \\
& \quad+\frac{1}{2 \pi i} \int_{\mathbb{C}} \partial_{\bar{z}} \tilde{g}_{0}(z, \tau) R_{n_{0}, A_{1}(\tau), g_{H}\left(x, D_{x}\right)}^{l}(z) d z \wedge d \bar{z}
\end{aligned}
$$

By the symbol calculus of PsDO, we have

$$
\left\|\operatorname{ad}_{A_{1}(\tau)}^{m}\left(g_{H}\left(x, D_{x}\right)\right)\right\|_{\mathfrak{B}(\mathbb{H})}=O(1) \quad \text { as } \quad \tau \rightarrow \infty \quad \text { for all } \quad 0 \leq m \leq n_{0} .
$$

The last term in the right hand side of (3.17) is dominated from above by

$$
\begin{aligned}
\int_{|z| \geq \epsilon^{\prime \prime} \tau} \tau^{-n_{0}-1}\left\langle\frac{z}{\tau}\right\rangle^{-3 / 2-n_{0}}|d z \wedge d \bar{z}| \cdot\left\|\operatorname{ad}_{A_{1}(\tau)}^{n_{0}}\left(g_{H}\left(x, D_{x}\right)\right)\right\| & \\
& =O\left(\tau^{1-n_{0}}\right)
\end{aligned}
$$

So it remains to prove that for $0 \leq m \leq n_{0}$

$$
g_{m}\left(A_{1}(\tau), \tau\right) f_{2}(K)\left(g_{\beta, \alpha, \epsilon}^{(1)}\left(A_{2}(\tau), \tau\right)\right)^{\frac{1}{2}} e^{-i \sigma K} f(K) B^{-\frac{\alpha}{2}}
$$

is square integrable. We apply (3.2) again to see that this is equal to

$$
\begin{aligned}
&\left\{\sum_{l=0}^{n_{0}-1} \frac{(-1)^{l}}{l !} \operatorname{ad}_{A_{1}(\tau)}^{l}\left(f_{2}(K)\right) g_{m}^{(l)}\right.\left.\left(A_{1}(\tau), \tau\right)+O_{\mathfrak{B}(\mathbb{H})}\left(\tau^{1-n_{0}}\right)\right\} \\
& \times\left(g_{\beta, \alpha, \epsilon}^{(1)}\left(A_{2}(\tau), \tau\right)\right)^{\frac{1}{2}} e^{-i \sigma K} f(K) B^{-\frac{\alpha}{2}}
\end{aligned}
$$

Here we note that $\tau^{1-n_{0}}\left(g_{\beta, \alpha, \epsilon}^{(1)}\left(A_{2}(\tau), \tau\right)\right)^{\frac{1}{2}}$ is square integrable with respect to $\tau$ because of the assumption (3.5) and the fact $\sup _{\tau \geq 1}\left\|\frac{A_{2}(\tau)}{\tau}\right\|_{\mathfrak{B}(\mathbb{H})}=L<$ $\infty$. Again using (3.2) we have

$$
\begin{aligned}
& g_{m+l}\left(A_{1}(\tau), \tau\right)\left(g_{\beta, \alpha, \epsilon}^{(1)}\left(A_{2}(\tau), \tau\right)\right)^{\frac{1}{2}} \\
&=\sum_{j=0}^{n_{0}-1} \frac{(-1)^{j}}{j !} \operatorname{ad}_{A_{1}(\tau)}^{j}\left(\left(g_{\beta, \alpha, \epsilon}^{(1)}\left(A_{2}(\tau), \tau\right)\right)^{\frac{1}{2}}\right) g_{m+l}^{(j)}\left(A_{1}(\tau), \tau\right) \\
& \quad+\frac{1}{2 \pi i} \int_{\mathbb{C}} \partial_{\bar{z}} \tilde{g}_{m+l}(z, \tau) R_{n_{0}, A_{1}(\tau), g^{(1)}}^{l}(z) d z \wedge d \bar{z}
\end{aligned}
$$


We rewrite $\left(g_{\beta, \alpha, \epsilon}^{(1)}(x, \tau)\right)^{\frac{1}{2}}$ as $\tau^{\frac{1}{2}(-\beta+\alpha-1)}\left(-\frac{x}{\tau}\right)^{\alpha / 2-1 / 2} \tilde{\chi}\left(\frac{x}{\tau}\right)$ and put

$$
h_{\tau}(x)=\tau^{\frac{1}{2}(-\beta+\alpha-1)}(-x)^{\alpha / 2-1 / 2} \tilde{\chi}(x) .
$$

Let $\rho(x) \in C_{0}^{\infty}(\mathbb{R})$ be real valued and satisfies $\rho(x) \equiv 1$ on $|x| \leq L+1$. By constructing an almost analytic extension of $h_{\tau}(x) \rho(x)$, which we denote by $\tilde{h}_{\tau}(z)$, we have

$$
\begin{aligned}
& \left(g_{\beta, \alpha, \epsilon}^{(1)}\left(A_{2}(\tau), \tau\right)\right)^{\frac{1}{2}}=\frac{1}{2 \pi i} \int_{\mathbb{C}} \partial_{\bar{z}} \tilde{h}_{\tau}(z)\left(z-\frac{A_{2}(\tau)}{\tau}\right)^{-1} d z \wedge d \bar{z} \\
& \operatorname{ad}_{A_{1}(\tau)}^{j}\left(\left(g_{\beta, \alpha, \epsilon}^{(1)}\left(A_{2}(\tau), \tau\right)\right)^{\frac{1}{2}}\right) \\
& \quad=\frac{1}{2 \pi i} \int_{\mathbb{C}} \partial_{\bar{z}} \tilde{h}_{\tau}(z) \operatorname{ad}_{A_{1}(\tau)}^{j}\left(\left(z-\frac{A_{2}(\tau)}{\tau}\right)^{-1}\right) d z \wedge d \bar{z}
\end{aligned}
$$

By induction, we can see that for $\operatorname{Im} z \neq 0$

$$
\left\|\operatorname{ad}_{A_{1}(\tau)}^{j}\left(\left(z-\frac{A_{2}(\tau)}{\tau}\right)^{-1}\right)\right\| \leq C_{j}|\operatorname{Im} z|^{-j-1},
$$

where $C_{j}$ is independent of $\tau$. Combining (3.24) and (3.25)

$$
\left\|\operatorname{ad}_{A_{1}(\tau)}^{j}\left(\left(g_{\beta, \alpha, \epsilon}^{(1)}\left(A_{2}(\tau), \tau\right)\right)^{\frac{1}{2}}\right)\right\|_{\mathfrak{B}(\mathbb{H})}=O\left(\tau^{(-\beta+\alpha-1) / 2}\right) \quad \text { as } \quad \tau \rightarrow \infty .
$$

Using (3.2) we compute

$$
\begin{aligned}
& g_{m+l}\left(A_{1}(\tau), \tau\right)\left(g_{\beta, \alpha, \epsilon}^{(1)}\left(A_{2}(\tau), \tau\right)\right)^{\frac{1}{2}} e^{-i \sigma K} f(K) B^{-\frac{\alpha}{2}} \\
&=O\left(\tau^{\frac{1}{2}(-\beta+\alpha-1)}\right) \sum_{j=0}^{n_{0}-1} g_{m+l}^{(j)}\left(A_{1}(\tau), \tau\right) e^{-i \sigma K} f(K) B^{-\frac{\alpha}{2}} \\
& \quad+O\left(\tau^{1-n_{0}+(-\beta+\alpha-1) / 2}\right) e^{-i \sigma K} f(K) B^{-\frac{\alpha}{2}}
\end{aligned}
$$

Here we apply (3.9) with $B=\left\langle A_{1}(1)\right\rangle^{1+\kappa}(\kappa>0)$. Then

$$
g_{m+l}^{(j)}\left(A_{1}(\tau), \tau\right) e^{-i \sigma K} f(K) B^{-\frac{\alpha}{2}}=O\left(\tau^{-\alpha(1+\kappa) / 2}\right)
$$

So we have proved

$$
\begin{array}{r}
\left(-\frac{A_{1}(\tau)}{\tau}\right)^{\frac{1}{2}} \chi\left(\frac{A_{1}(\tau)}{\tau}\right) g_{H}\left(x, D_{x}\right) f_{2}(K)\left(g_{\beta, \alpha, \epsilon}^{(1)}\left(A_{2}(\tau), \tau\right)\right)^{\frac{1}{2}} e^{-i \sigma K} \\
f(K) B^{-\frac{\alpha}{2}} \\
=O\left(\tau^{-\frac{1}{2}-\frac{\alpha \kappa}{2}}\right)
\end{array}
$$

is square integrable in $\tau$. 
Hence the conclusions of Theorem 3.4 and Corollary 3.5 hold. i.e.

$$
\left\|\chi\left(\frac{A_{2}(\tau)}{\tau}\right) e^{-i \sigma K} f(K)\langle A\rangle^{-s^{\prime}}\right\|_{\mathfrak{B}(\mathbb{H})}=O\left(\tau^{-s}\right)
$$

for all $0<s<s^{\prime}$ as $\tau \rightarrow \infty$

Our final aim is to change the weight in (3.29) by functions of $x$.

Proof of Theorem 1.6. It follows from (3.29) that

$$
\left\|\chi\left(\frac{A_{2}(\tau)}{\tau}<-\epsilon\right) e^{-i \sigma K} f(K)\langle x\rangle^{-s^{\prime}}\right\|_{\mathfrak{B}(\mathbb{H})}=O\left(\sigma^{-s}\right) \quad \text { as } \quad \sigma \rightarrow \infty
$$

Therefore Theorem 1.6 is proved if we show for any $N \in \mathbb{N}$,

$$
\begin{aligned}
\chi\left(\frac{|x|^{2}}{4 \tau^{2}}\right. & \left.-\frac{d(I, \mathbb{Z})}{d(I, \mathbb{Z})+1}<-\epsilon\right) \chi\left(\frac{A_{2}(\tau)}{\tau}<-\epsilon\right) \\
& =\chi\left(\frac{|x|^{2}}{4 \tau^{2}}-\frac{d(I, \mathbb{Z})}{d(I, \mathbb{Z})+1}<-\epsilon\right)+O_{\mathfrak{B}(\mathbb{H})}\left(\tau^{-N}\right) \quad \text { as } \quad \sigma \rightarrow \infty .
\end{aligned}
$$

Again we use an almost analytic extension of $\chi \rho($ denoted by $\tilde{\chi})$ and

$$
\chi\left(\frac{A_{2}(\tau)}{\tau}\right)=\frac{1}{2 \pi i} \int_{\mathbf{C}} \partial_{\bar{z}} \tilde{\chi}(z)\left(z-\frac{A_{2}(\tau)}{\tau}\right)^{-1} d z \wedge d \bar{z} .
$$

We denote the symbol of $\frac{A_{2}(\tau)}{\tau}$ as $a_{\tau}(x, \xi)$.

Then

$$
R_{\tau}(x, \xi)=a_{\tau}(x, \xi)-\frac{1}{\tau} g(-\tau M, \tau) \in S\left(\tau^{-1}\langle\xi\rangle^{-1}\right)
$$

We construct a parametrix of $\left(z-\frac{A_{2}(\tau)}{\tau}\right)$ by putting

$$
\left\{\begin{aligned}
q_{0}(x, \xi) & =\left(-\frac{1}{\tau} g(-\tau M, \tau)+z\right)^{-1} \\
q_{j}(x, \xi) & =-\sum_{\substack{j^{\prime}+|\alpha|=j \\
j^{\prime}<j}} \frac{1}{\alpha !}\left(-\frac{1}{\tau} g(-\tau M, \tau)+z\right)^{(\alpha)} q_{j^{\prime}(\alpha)} q_{0} \\
& -\sum_{j^{\prime}+|\alpha|=j-1} \frac{1}{\tau} R_{\tau}^{(\alpha)} q_{j(\alpha)} q_{0} \quad(j \geq 1)
\end{aligned}\right.
$$

Then

$$
\left(z-\frac{A_{2}(\tau)}{\tau}\right) \sum_{j=0}^{N} q_{j}\left(x, D_{x}\right)-\mathbf{I} \in \mathrm{Op} S\left(\tau^{-N}\right) .
$$


Moreover we have the following estimates: There exists $l \gg 1$ such that

$$
\left\|\left(z-\frac{A_{2}(\tau)}{\tau}\right) \sum_{j=0}^{N} q_{j}-\mathbf{I}\right\|_{\mathfrak{B}(\mathbb{H})} \leq C \tau^{-N}|\operatorname{Im} z|^{-N-l}
$$

So replacing the resolvent by the parametrix $\sum q_{j}\left(x, D_{x}\right)$ we have

$$
\begin{aligned}
\chi\left(\frac{A_{2}(\tau)}{\tau}\right)= & \frac{1}{2 \pi i} \int_{\mathbb{C}} \partial_{\bar{z}} \tilde{\chi}(z)\left(z-\frac{A_{2}(\tau)}{\tau}\right)^{-1} d z \wedge d \bar{z} \\
= & \sum_{j=0}^{N} \frac{1}{2 \pi i} \int_{\mathbb{C}} \partial_{\bar{z}} \tilde{\chi}(z) q_{j}\left(x, D_{x}\right) d z \wedge d \bar{z} \\
& \quad+\tau^{-N} \int_{\mathbb{C}} \partial_{\bar{z}} \tilde{\chi}(z) O\left(|\operatorname{Im} z|^{-N-l-1}\right) d z \wedge d \bar{z}
\end{aligned}
$$

Combined with the fact that

$$
\chi\left(E^{\prime \prime}-\epsilon>\frac{|x|^{2}}{4 \tau^{2}}\right) \chi\left(-g\left(-\tau M\left(\frac{x}{\tau}, \xi\right), \tau\right) / \tau\right)=\chi\left(E^{\prime \prime}-\epsilon>\frac{|x|^{2}}{4 \tau^{2}}\right)
$$

this shows

$$
\begin{aligned}
& \chi\left(E^{\prime \prime}-\epsilon>\frac{|x|^{2}}{4 \tau^{2}}\right) \chi\left(\frac{A_{2}(\tau)}{\tau}\right) \\
& \quad=\chi\left(E^{\prime \prime}-\epsilon>\frac{|x|^{2}}{4 \tau^{2}}\right)+O_{\mathfrak{B}(\mathbb{H})}\left(\tau^{-N}\right) \text { as } \quad \tau \rightarrow \infty
\end{aligned}
$$

Since $N$ is arbitrary, we take $N>s$ and obtain Theorem 1.6.

\section{REFERENCES}

[Gé1] C. Gérard, Sharp propagation estimates for $N$-particle systems, Duke Math. J., 67 (1992), 483-515.

[Gé2] C. Gérard, Asymptotic completeness for 3-particle long-range systems, Invent. Math., 114 (1993), 333-397.

[H-S] B. Helffer and J. Sjöstrand, Equation de Schrödinger avec champ magnétique et équation de Harper, Lecture Notes in Physics, 345, Schrödinger Operators, (1989), Springer, Berlin-Heidelberg-New York, 118-197.

[How] J. Howland, Scattering Theory for Hamiltonians Periodic in Time, Indiana Univ. Math. J., 28 (1979), 471-494.

[Ka] T. Kato, Wave operators and similarity for some nonselfadjoint operators, Math. Ann., 162 (1966), 258-276. 
[Ki-Y] H. Kitada and K. Yajima, A scattering theory for time-dependent long range potentials, Duke Math. J., 49 (1982), 341-376.

$[\mathrm{Ku}-\mathrm{Y}]$ Y. Kuwabara and K. Yajima, The limiting absorption principle for Schrödinger operators with long-range time-periodic potentials, J. Fac. Sci. Univ. Tokyo Sect. IA, Math., 34 (1987), 833-851.

[Mo] E. Mourre, Absence of singular continuous spectrum for certain self-adjoint operators, Commun. Math. Phys., 78 (1981), 391-408.

[Na] S. Nakamura, Asymptotic completeness for three-body Schrödinger equations with time periodic potentials, J. Fac. Sci. Univ. Tokyo Sect. IA, Math., 33 (1986), 379-402.

[PSS] P. Perry, I. M. Sigal and B. Simon, Spectral analysis of N-bodySchrödinger operators, Ann. Math., 114 (1981), 519-567.

[R-S] M. Reed and B. Simon, Methods of Modern Mathematical Physics, 1-4, Academic Press, New York-San Francisco-London.

[Sk] E. Skibsted, Propagation estimates for $N$-body Schrödinger operators, Commun. Math. Phys., 91 (1991), 67-98.

[Ya] K. Yajima, Scattering theory for Schrödinger operators with potentials periodic in time, J. Math. Soc. Jpn., 29 (1977), 729-743.

Department of Mathematics

Osaka University

Toyonaka 560-0043

Japan

yokoyama@math.sci.osaka-u.ac.jp 